\title{
Image Analysis of Microfluidics: Visualization of Flow at the Microscale
}

\author{
Dr. Michael G Mauk P.E., Drexel University \\ Dr. Richard Chiou, Drexel University (Eng.) \\ Dr. Vladimir Genis, Drexel University (Tech.) \\ Mr. Eric Carr, Drexel University
}

Mr. Eric Carr is currently the laboratory technician for Drexel University's Engineering Technology program. Carr assists faculty members with the development and implementation of various Engineering Technology courses, and enjoys finding innovative ways to use microcontrollers and other technologies to enhance Drexel's Engineering Technology course offerings. Eric holds an M.S. in Computer Engineering from Drexel University and is the author of several recent technical papers in the field of Engineering Technology Education. 


\title{
Image Analysis of Microfluidics: Visualization of Flow at the Microscale
}

\begin{abstract}
Microfluidics is the study and application of fluid flow at the microscale. As a representative example, many microfluidic devices and systems are based on a polymer substrate ('chips') in which a miniaturized fluidic network of channels, conduits, chambers, filters, packed beds, valves, and fluid actuators is fabricated using various prototyping methods. Feature sizes (e.g., channel widths) range from 0.1 to several millimeters. Fluid actuation and flow control can be effected by a number of different mechanisms to implement a process comprised of metering, heat transfer, mixing, reaction, and separation steps, monitored with sensors such as pressure gauges, thermocouples, RTDs, and photodetectors. Furthermore, fluid flow in the chips is highly amenable to image capture, processing, and analysis using visible and thermal (infrared) cameras. The interdisciplinary nature of microfluidics, its relative low cost and accessible technology, and its importance in emerging technologies suggest a wider use of microfluidicsbased systems in engineering education. Our aims are to develop a series of microfluidics-based experiments to demonstrate concepts in fluid mechanics and heat/mass transfer, as well as develop student skills in CAD, prototyping, sensors, instrumentation and control, and image analysis. Chips can be designed, fabricated, and operated in time frames of hours, and such projects provide students with an opportunity for conceptualization, design, prototyping, implementation, and analysis of fluidic and thermal systems in a readily accessible and easilyinterpretable format. Here we report approaches and techniques to provide resources for instruction and experimentation in microfluidics-based fluid mechanics, with emphasis on characterizing various flow modes with image capture, processing, and analysis.
\end{abstract}

\section{Introduction and Background}

Microfluidics is the science and technology of miniaturizing fluidic systems for implementing chemical and biological processes on a microscale, i.e., fluid flow in channels or conduits with cross-sectional dimensions of 1 micron to 1 millimeter. Application areas include analysis and synthesis of materials, biomedical diagnostics, biotechnology, and as platforms for nanotechnology. Microfluidics also serves an effective vehicle for studying and teaching various physical and chemical phenomena important in engineering science, and for developing and integrating skills in diverse engineering subjects such as CAD, prototyping, materials, fluid mechanics, heat and mass transfer, chemical kinetics, spectroscopy, micro-optics, sensors, instrumentation and control, microcontrollers, machine vision, thermal imaging, and computational fluid mechanics. Microfluidic systems can be realized in formats that are amenable to common rapid prototyping (CNC and laser machining, 3-D printing) and microfabrication (optical and soft lithography in PDMS), and such systems can broadly utilize microelectronics, microelectromechanical systems (MEMS), fiber optics, miniature sensors, and microcontroller- and LabVIEW ${ }^{\text {TM}}$-based instrumentation, as well as image capture and image 
analysis. We are developing microfluidic-oriented laboratory projects for teaching topics in prototyping, fluid mechanics, chemical kinetics, electrochemistry, separation science, heat and mass transfer, process control, biotechnology, materials science, and image processing. Microfluidicis is also an useful gateway for introducing engineering students to biotechnology and biomedical fields, since many chemical and biotechnology processes done on the chemistry lab bench can be translated to microfluidic formats. Moreover, microfluidics is emerging as an important commercial technology, and engineering students will be well served by gaining knowledge and experience in this field.

A typical microfluidic system is centered around a credit-card sized plastic 'lab on a chip' that hosts a fluidic network of channels, conduits, manifolds, inlet/outlet ports, chambers, and may also include filters, packed beds, valves, integrated sensors (thermocouples or RTDs, and fluidic actuators. Channel dimensions are typically about $1 \mathrm{~mm}$ or less in cross-section. Plastics commonly used for chip fabrication include acrylic (PMMA), polycarbonate, and polystyrene. The plastic material is optically transparent allowing image capture of the fluid flow in the chip. The chips can be prototyped using a CO2 laser cutter, a CNC machine, 3-D printer, or other types of sheet cutting tools.

As fluids flow through the transparent plastic chip, the flow and operational characteristics of the chip can be visualized with a CCD camera mounted over the chip. The visualization of flow is aided by using colored or fluorescent dyes. A thermal camera can also be used to map temperature profiles where heating and cooling effects are important.

In the following, we describe some of the experiments we are developing that use microfluidic chips as projects and case studies for undergraduate engineering technology. These are incorporated into current traditional courses on fluid mechanics, engineering measurements, heat transfer, prototyping, robotics, and microcontrollers, as well as capstone Senior Design projects. The advantages of microfluidics-based experiments for instructional purposes include modest equipment costs, small space requirements (all of the experiments can be done on a table top), miniscule generation of waste products, and varying levels of sophistication as appropriate for different student audiences. The principles and operation of the chip are readily apparent and conducive to visualization by several approaches, such as with CCD cameras and recently available low-cost thermal (IR) cameras. For the experiments described here and similar projects, students can design, prototype, instrument and operate the chips, and collect and analyze data, in time frames of several hours. A broad objective of this work is to foster students to think holistically about all aspects of the microfluidic system, and further appreciate the interrelationships between the fluidic, thermal, and optical aspects of the systems, and the role sensors, microcontrollers, and imaging cameras can play in the control and analysis of the systems.

Many microfluidics experiments can be done on a desktop or small section of a lab bench. Power requirements and materials consumption are minimal, as are safety concerns and 
generation of waste products. The small-scale of microfluidics is advantageous for sustainable education projects where schools may have limited resources to invest in conventional laboratory equipment. Further, microfluidics is part of a general trend in miniaturization technology that includes microelectronics, micro-optics, MEMS (micro electro mechanical systems), nanotechnology, and various sensors. Students should develop intuition about the scaling down engineering systems for improved performance, portability, lower cost, lower power requirements, reduced environmental impact, improved sustainability and more appropriate technologies for the developing world.

Our objective is to develop a set of microfluidics-based experiments and projects that can be integrated into undergraduate science and engineering curricula to support educational objectives in a number of courses. Thus, these experiments can replace or supplement current laboratory activities to provide coverage of traditional topics, while leveraging features of microfluidics to simultaneously introduce students to allied topics of contemporary and increasing interest.

\section{Chip Design and Fabrication}

There are many options for the materials and fabrication of microfluidic chips, as well as a multitude of approaches for implementing valves, actuators, and other means of flow control. In contrast to semiconductor microelectronics technology — where there is a broad consensus on the types of materials (silicon, silicon dioxide, GaAs) and processes (chemical vapor deposition, vacuum thin-film deposition, thermal oxidation, photolithography, etc.) that are best suited for electronic devices and fabrication - the microfluidics field remains "wide open” with regard to the selection of materials (soft or hard polymers, glass, silicon), fabrication methods (photolithography, soft lithography, laser cutting, CNC milling, 3-D printing, injection molding, stamping or embossing, etc.. ), and varied implementations of flow control and actuation devices such as valves and pumps. In microfluidics, many issues related to engineering design and fabrication approaches for realizing systems and devices are still unsettled. Students can find this situation either frustrating or intriguing as there are many opportunities for creative and novel approaches in this emerging field. The sophistication of the chip itself a fundamental design consideration. For example, to implement flow control, specifically the use of valves and fluid actuators either built into the chip (i.e., integrated) or external to the chip as supporting equipment (e.g., syringe pump or selector valve), and their particular implementation presents a multitude of options. At one extreme, chip comprises a passive fluidic device with a fluidic circuit where all fluidic actuation, flow control, heating/cooling, and sensors are external to the chip. This chip is easy to fabricate and relies on peripheral components such as thermoelectric elements, At the other extreme, an autonomous chip can include integrated valves, heating/cooling elements, and sensors, with

The chips are designed with AutoCAD ${ }^{\mathrm{TM}}$ or SolidWorks ${ }^{\mathrm{TM}}$. The chips are fabricated as laminates. A middle layer is machined with through cuts to define the fluidic circuit in a thin (1 $\mathrm{mm}$ ) sheet of plastic using a laser cutter or CNC machine. The channels and chambers of the 
middle layer are enclosed by attaching top and bottom plastic sheet layers using thermal bonding, solvent bonding, adhesives, or special double-sided tapes. Samples and reagents can be pipetted directly into the chip or extracted from the chip through inlet/outlet ports.

Alternatively, fluidic interconnections are provided by small-diameter (Teflon) tubing inserted through sidewall ports of the chip. We also found disposable pipette tips (e.g., Denville Sharp ${ }^{\mathrm{TM}}$ precision barrier tips), shorted by cutting off the tip and with fibrous plug removed, made good transitions between tubing and chip ports (drilled holes). The tubing and sideport hole diameters are selected to make a snug, leak-tight fit. Using tubing interconnections, the liquids can be delivered to the fluidic circuit with a pipette or a syringe, actuated either manually or with a programmable syringe pump.
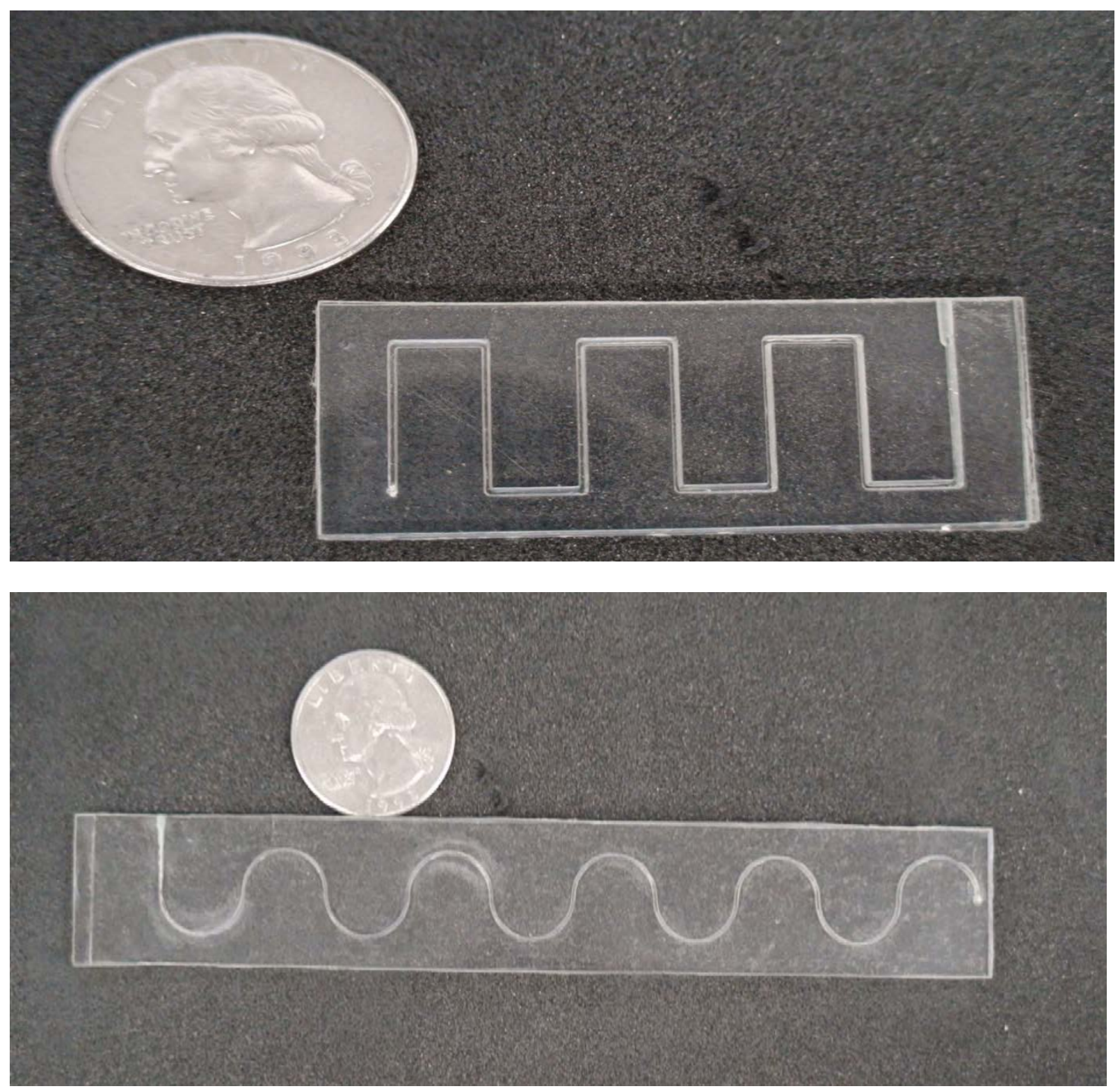

Figure 1.... 

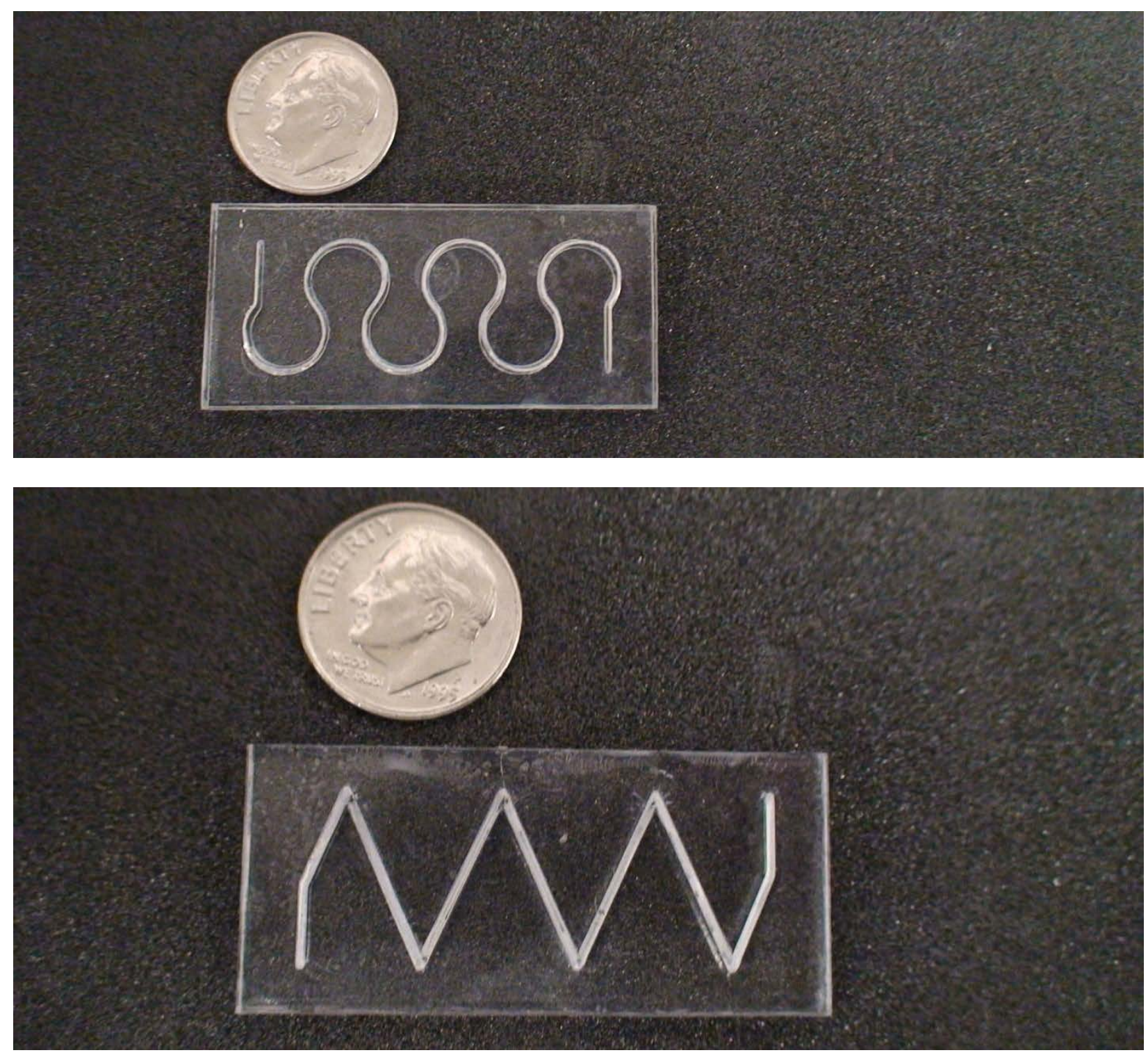

Figure 1: Some microfluidic chips for flow studies. These chips comprise three bonded layers acrylic sheet where the channel is cut in the middle laser with a $\mathrm{CO} 2$ laser machine. The chips are bonded with acrylic cement (TAP ${ }^{\circledR}$ acrylic cement). Top: rectangular flow path; top middle: serpentine flow path. Bottom middle: serpentine channel with tighter loops; bottom: triangular flow path. 


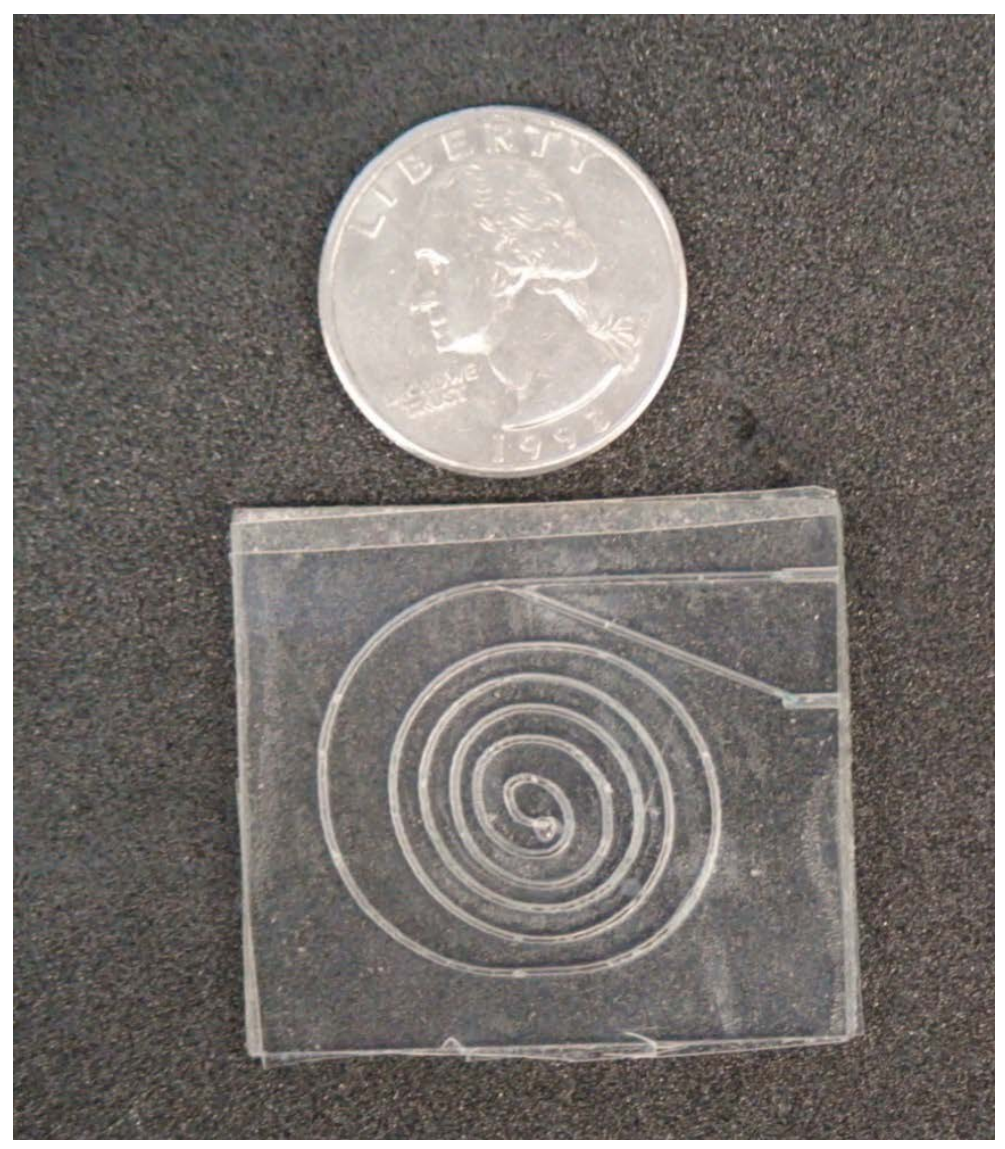

Figure 2: Spiral microfluidic circuit with two inputs to show mixing effects.

\section{Sensors, Instrumentation and Interfacing}

For temperature measurements, a small-diameter thermocouple wire (e.g., Type K) can be attached to the outside of the chip over a channel at the point where temperature of the fluid is to be measured. For improved accuracy, the thermocouple can also be bonded in between the acrylic sheets during chip assembly so it is in direct contact with the flow channel, but then the thermocouple is not easily reusable. RTDs can also be used to measure temperature.

Temperature precisions of $\pm 0.5^{\circ} \mathrm{C}$ are attainable. A simple non-contact measurement can also be done with a pocket IR thermometer (e.g., Radioshack 22-17). We are currently exploring the use of thermal imaging cameras for quantitative temperature studies of microfluidic devices.

For pressure measurements, we use silicon membrane transducers (SensorTechnics, First Sensor AG, Munich, Germany) that produce a voltage/pressure signal with a sensitivity of 240 $\mu \mathrm{V} / \mathrm{mbar}$ ( 0 - 50 mbar pressure range) to $10 \mu \mathrm{V} / \mathrm{mbar}$ ( 0 - 1 bar pressure range).

Localized heating and cooling of fluids in channels and chambers is effected with a small Peltier cooler that operates with about $3.5 \mathrm{~V}, 0$-3 A dc power. 
We imaged fluid flow in the chip during its operation with a small palm-sized digital CCD microscope (Aven Mightyscope ${ }^{\mathrm{TM}}$ ) mounted over the chip. The camera plugs into the USB port of a PC and produces videos (30 frames/s) of fluid flow. The magnification ranges from 10X to $500 \mathrm{X}$. Other camera types and set-ups are of course workable as well, including higher-end consumer CCD cameras. The quantification of flow rate can be made adding a graduated scale in the image, such as with a thin plastic ruler placed along the flow channel which shows the position of the flow front in each frame along with the time for that frame. From this analysis, the flow velocity can be accurately determined as a function of time. A common analysis objective is to determine the pressure drop $\Delta P$ between two points as a function of flow rate $Q$ or fluid velocity $v$.

$$
\Delta P=f(Q, \text { channel parameters })
$$

Figure 3 shows our typical setup for video imaging fluid flow in microfluidic chips. The chip is placed in the field of view of the camera and a video of the flow progression from the inlet port to the outlet port is captured. 


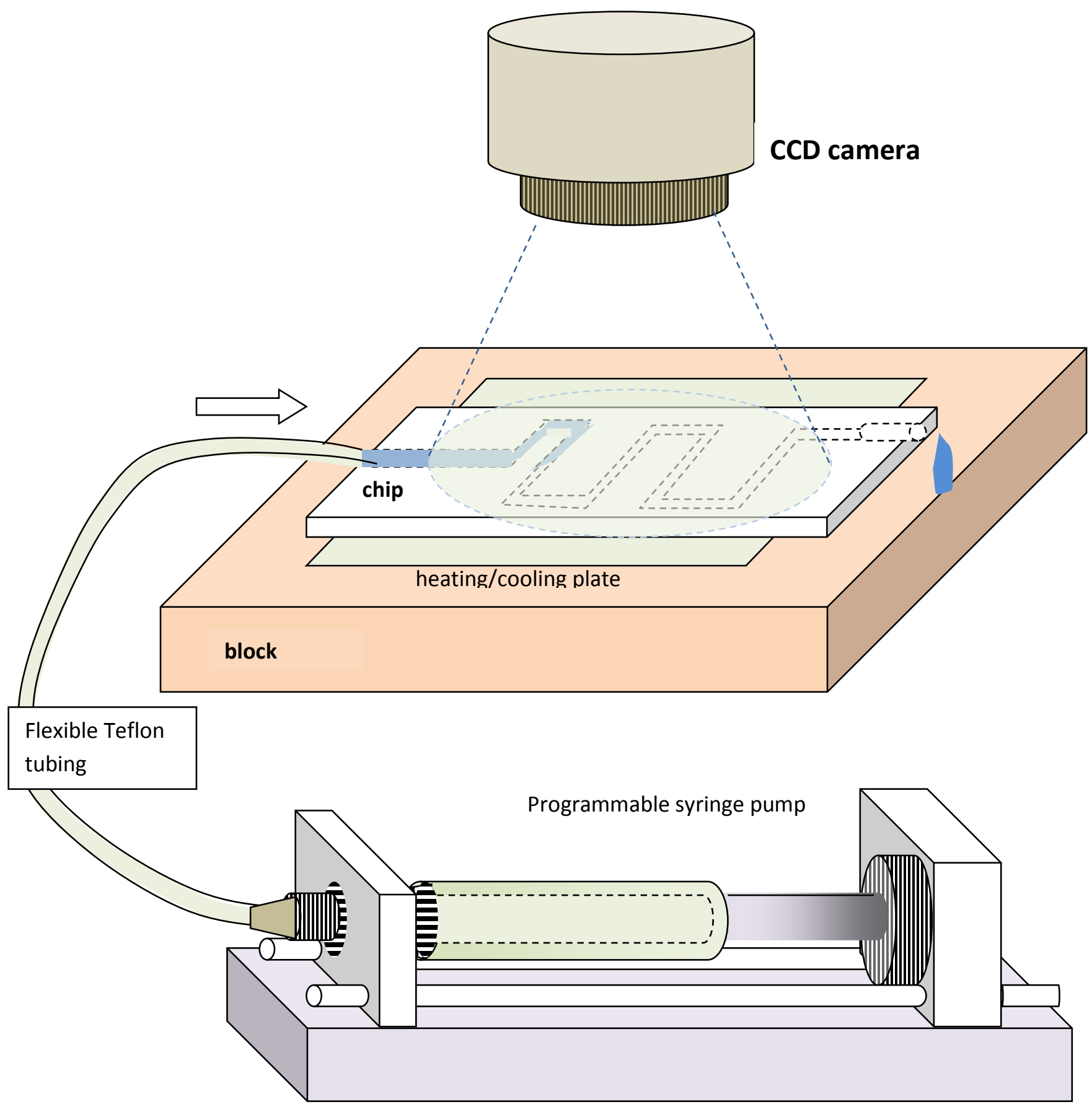

Figure 3: Syringe-pump drive and chip stage with CCD camera. 
The chip channel has side holes into which are inserted pipette tips to couple the pressure transducers to the channel (Figure 4). Another interesting and important flow type is related to flow through porous media. Various filter paper or other fibrous media (e.g., Whatman glass fibers, cellulose, nitrocellulose, Porex ${ }^{\mathrm{TM}}$ ) can be packed into a chamber on the chip prior to bonding (Figure 5). The flow front of a dyed liquid for different packed bed geometries can be directly visualized.

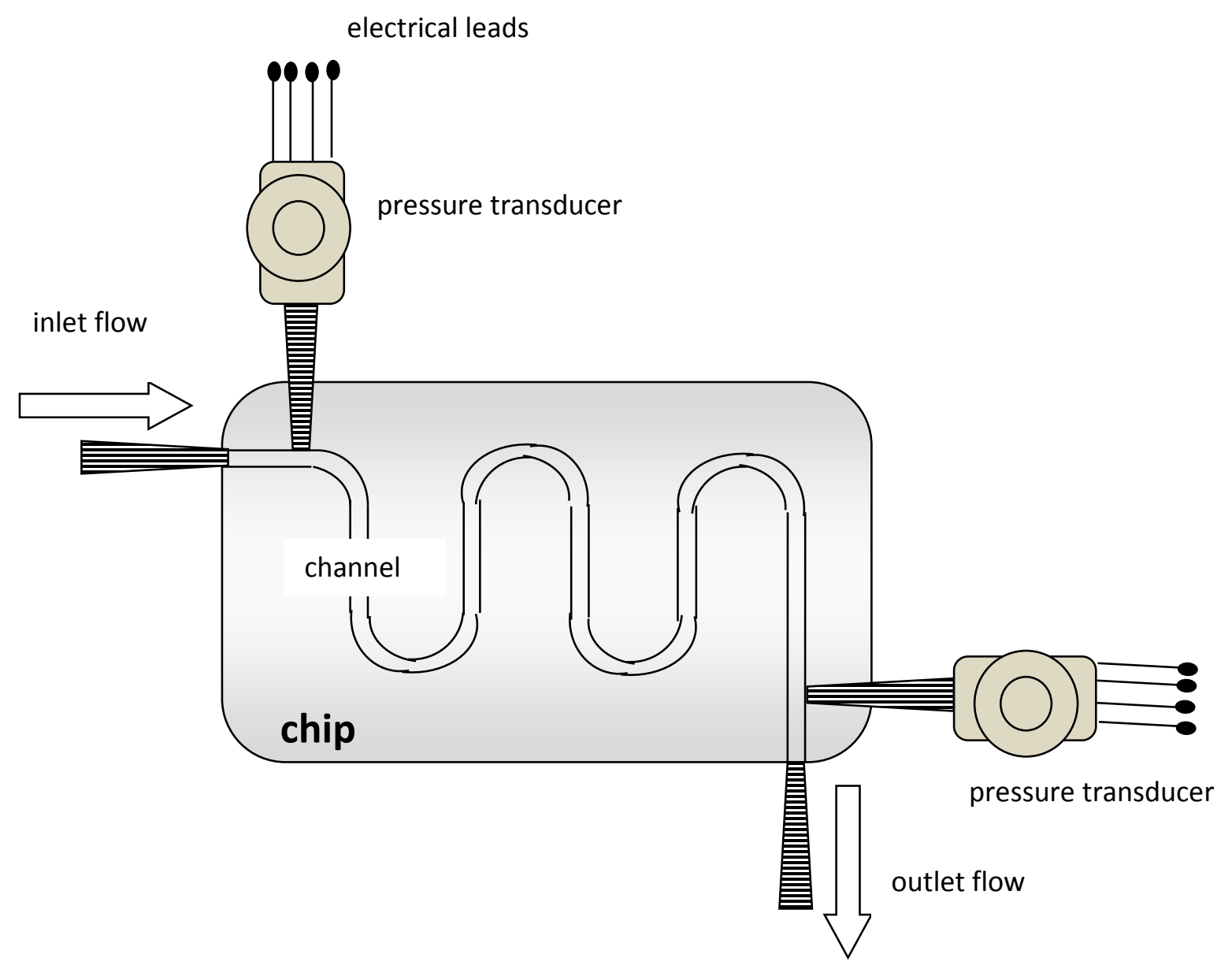

Figure 4: Siting of pressure transducers. (Top view of chip). 


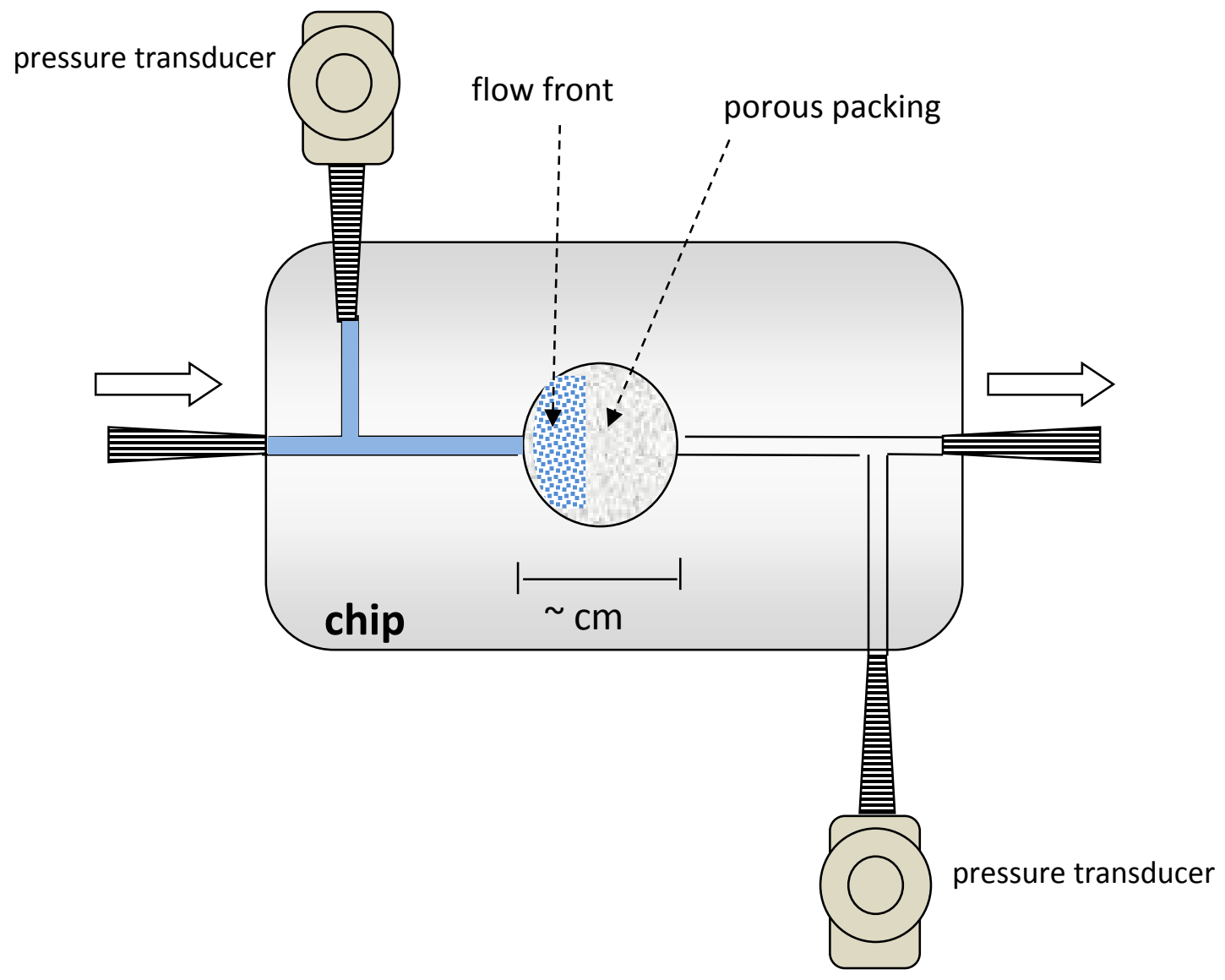

Figure 5: Chip to measure flow characteristics through porous media (Top view of chip).

We also developed chips to illustrate heating and 'phase transition' phenomena (Figure 6). A chip with a chamber is filled with a water-based suspension of Expancel ${ }^{\mathrm{TM}}$ microspheres (www.akzonobel.com). These hydrocarbon-filled polymer beads irreversibly expand from 12 microns diameter to 40 microns diameter when heated to about $80^{\circ} \mathrm{C}$, creating a high pressure in the chamber. Heating options include lights, hot air guns, thermoelectric heaters or resistive heaters. The response (increase in measured pressure) to various heating programs can be characterized as a case study in systems analysis. 


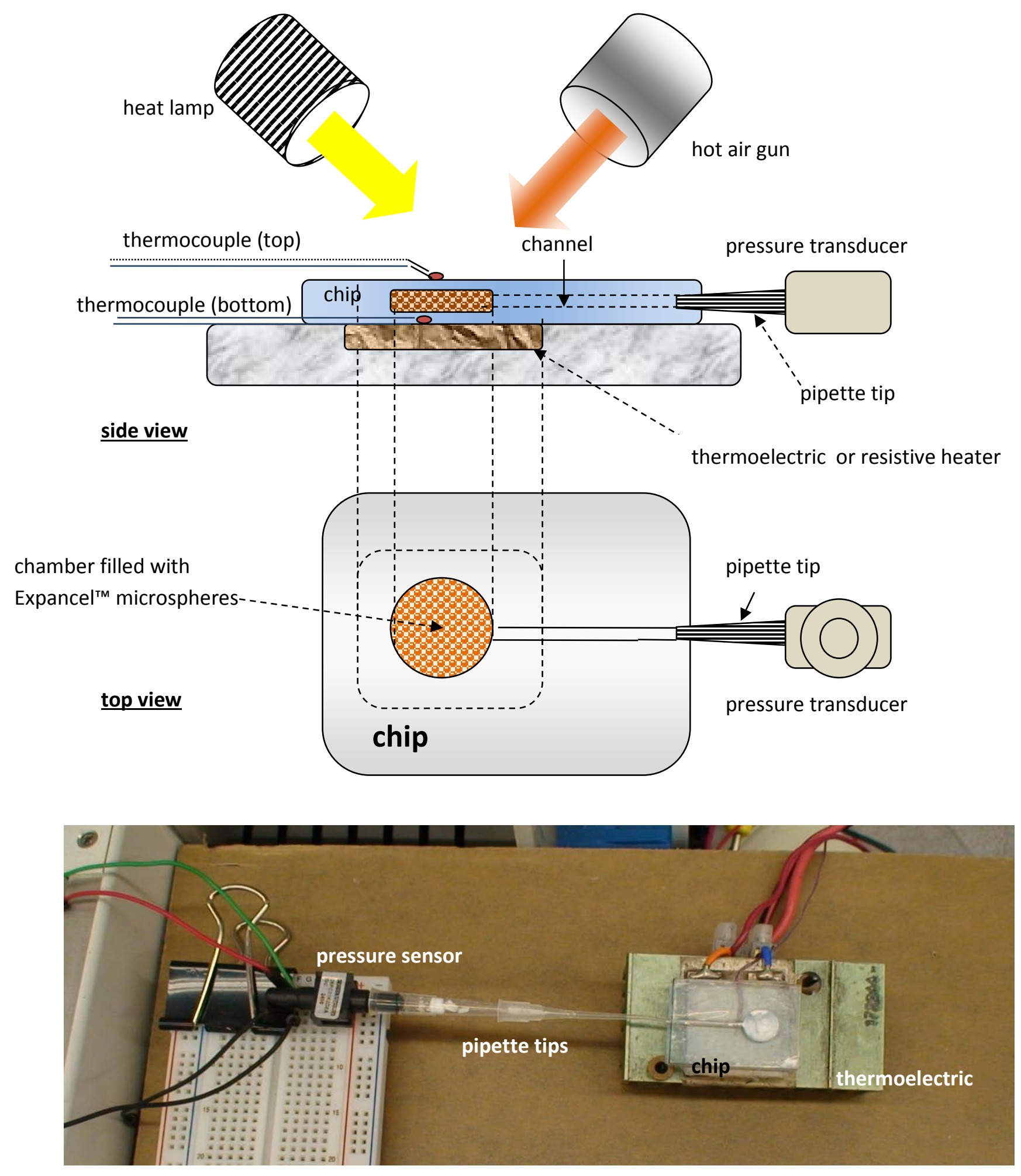

Figure 6: Chip to measure pressure transients due to heating of hydrocarbon-filled polymer microspheres (Expancel ${ }^{\mathrm{TM}}$ ). 


\section{Flow Driving Forces}

Characterization of flow in microfluidic channels of various shapes and dimensions and for different types of driving forces that can include:

Hydraulic flow: in syringe driven flow, this is flow driven by displacement of fluid due to movement of the syringe plunger. The flow rate is proportional to the speed of the motorized plunger.

Pneumatic flow: in syringe-driven flow, the syringe contains a charge of air which is compressed to create a pressure head on the liquid in the channel.

Capillary flow: In capillary flow, a quantity of liquid is added to the inlet port with a pipette. Surface tension forces wick the liquid into the channel. The surface tension of the liquid can be modified by adding surfactants such as Tween ${ }^{\mathrm{TM}}$ or sodium dodecyl sulfate (SDS).

Gravitational Flow: The chip can be tilted out of the horizontal plane so that gravitational forces supplement or diminish other operative driving forces.

Magnetic Forces: We used a ferrofluid in the channels and an external magnet to push the ferrofluid through the channels. 


\section{Image Analysis of Microfluidic Flow}

The frames a video are digitized and used to model the kinematics of flow in the microchannels. Figure 7a show a frame from the video of blue-dyed water pumped through a serpentine channel (width $0.9 \mathrm{~mm}$ ). Figure $\mathbf{7 b}$ shows the frame bitmap processed according to the steps listed below.

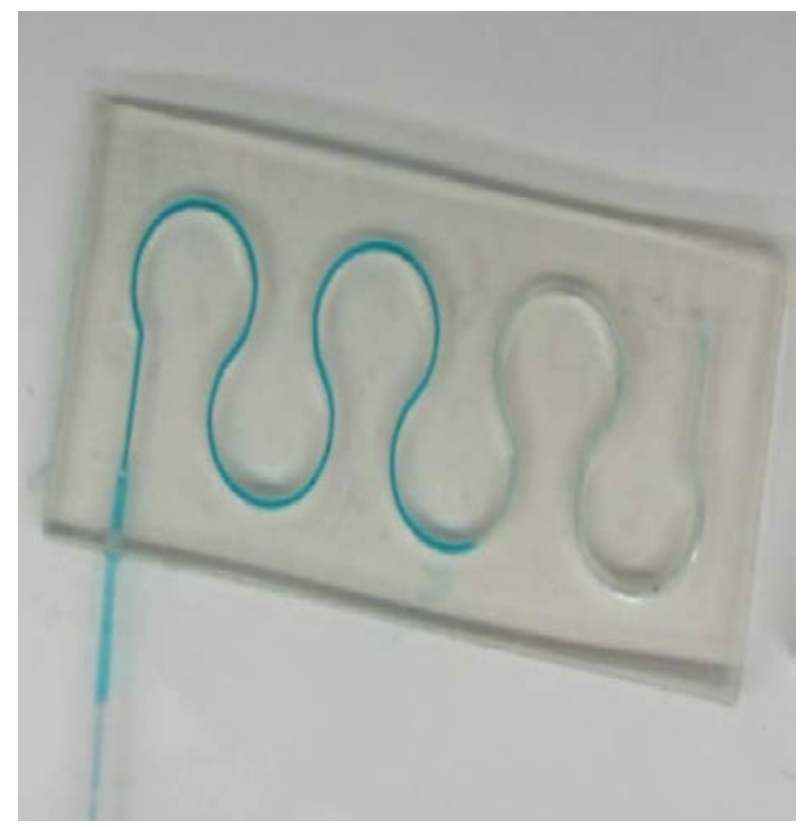

Figure 7a: Frame from original captured video of blue-dyed water pushed by programmable syringe pump through serpentine channel of microfluidic chip.

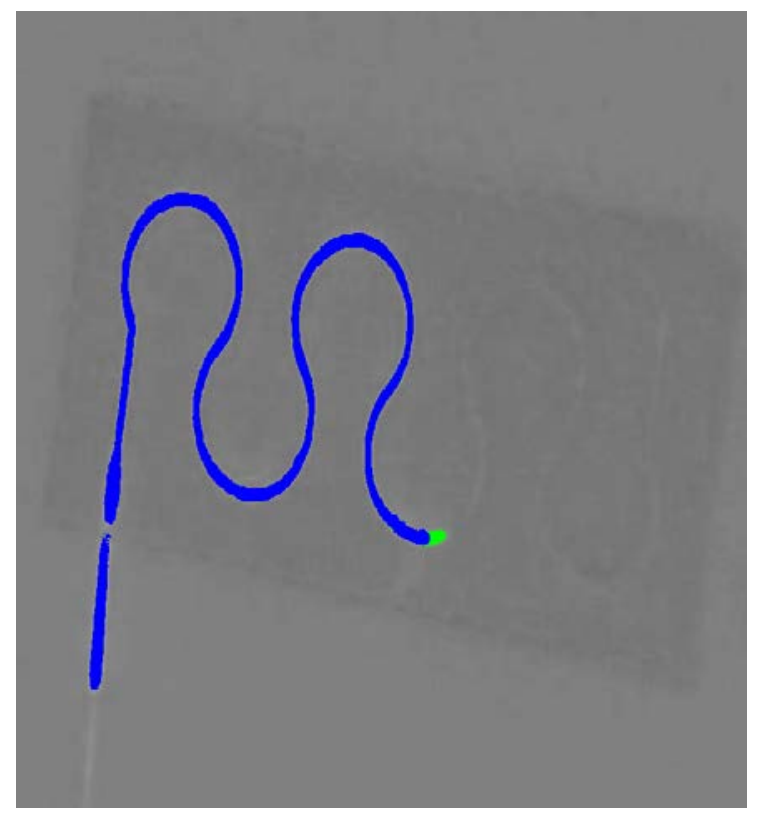

Figure 7b: Bitmap of frame processed to threshold blue bits corresponding to blue-dyed fluid in channel.

Frames from the Microsoft Windows media player (color) video are digitized as bit maps, and processed according to the following steps:

- $\quad$ Each pixel is converted to a "blueness" value: blueness $=b /(r+g)$; if $(r+g)=0$ then blueness $=b$, where $r, b$, and $g$ are the tricolor (red, blue, and green) pixel intensities.

- $\quad$ In each frame, pixels which increase $19 \%$ (this seems to work best) in blueness over the previous frame are marked

- $\quad$ Marked pixels which were not already marked are shown in green as "new" flow

- $\quad$ Pixels which were already marked (including last frame’s green pixels) are marked in blue as "old" flow.

- $\quad$ All other pixels are shown in grayscale, where brightness = blueness times a correction factor. 
The total number of blue pixelsin a frame is an indicator of the volume of (blue-dyed) fluid in the channel at the corresponding flow time of the frame. For a syringe pump driven flow, the volume is expected to be a linear function of time (or number of frames). The total number of pixels as a function of frame number is plotted in Figure 8. Similar plots for capillary flow showed the expected $\sqrt{t}$ dependence.

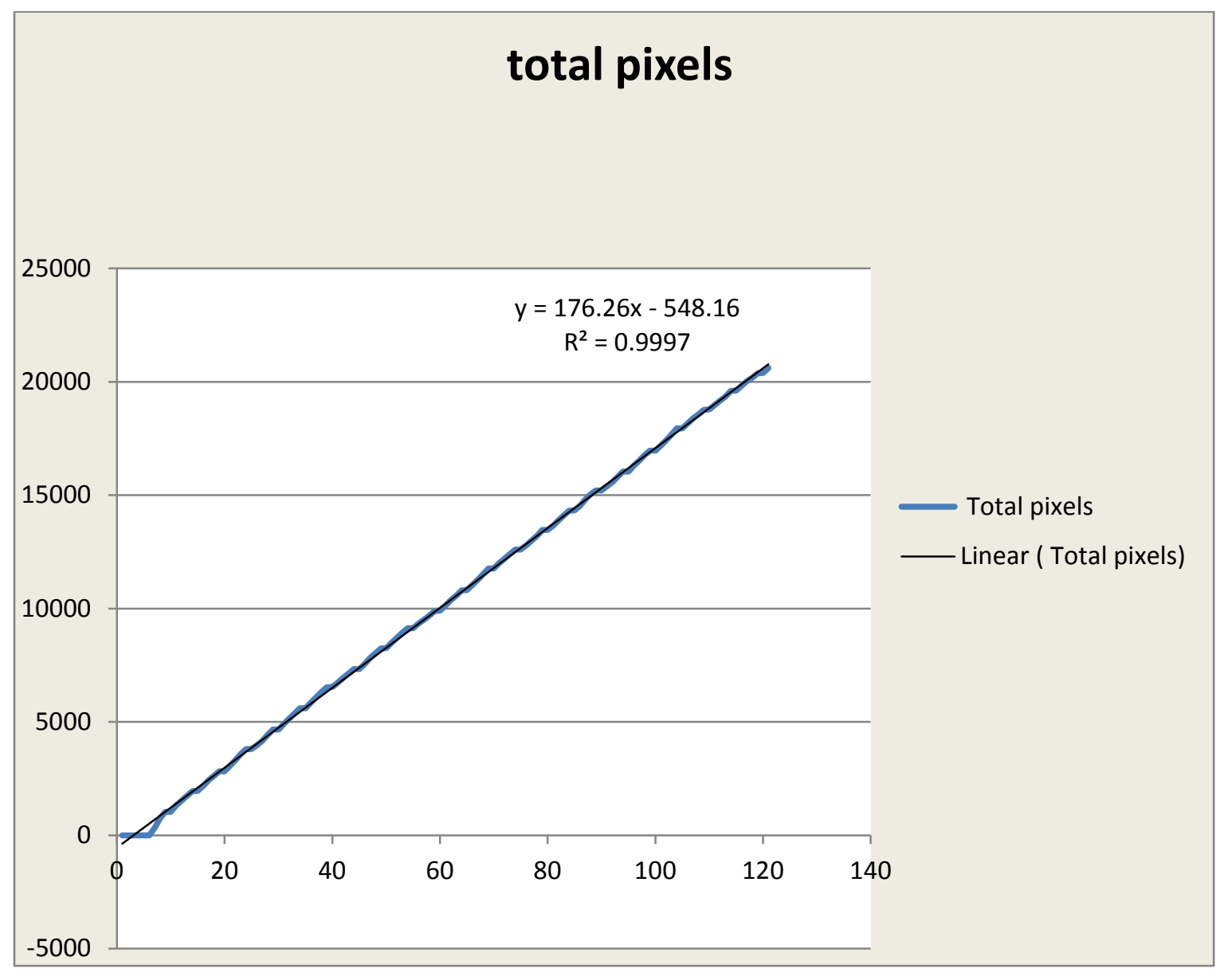

Figure 8: Plot of total pixels as a function of frames (30 frames/sec) showing linear relationship between volume and time. 


\section{Discussion and Conclusion}

Microfluidic devices can be prototyped and instrumented to study fluid flow and thermal phenomena. A microfluidic 'chip' can host many types of fluidic circuits, i.e., networks of channels, chambers, conduits, packed beds, and the like which exhibit varied paradigms of fluidic and thermal phenomena, and moreover, that are representative of typical 'lab on a chip' applications. Microfluidic devices, made in clear plastic and measuring several centimeters on a side with flow channel features on the order of $0.1 \mathrm{~mm}$ to several $\mathrm{mm}$, loaded with dyed liquids, are well-suited for image capture and subsequent image processing and analysis in order to characterize the flow. For instructional purposes, the approaches described here offer a useful means for visualization of flow. The design and fabrication of the microfluidic chips and systems are instructive projects due to their highly interdisciplinary nature. Microfluidics based experiments allow students to work from design through prototype to test with modest resources and time requirements.

Acknowledgement: This work was supported by National Science Foundation (Phase 1) TUES Grant 1044708. 


\section{References and Bibliography}

H. Becker and C. Gartner [2008], "Polymer fabrication technologies for microfluidic systems" Anal Bioanal Chem. 390(1) 89-111.

B.J. Beebe, G.A. Mensing, and G.M. Walker [2002], "Physics and Applications of Microfluidics in Biology” Annual Review of Biomedical Engineering 4: 261-285.

F. Caiazzo, F. Curcio, G. Daurelio, F. Memola Capece Minutolo [2009], "Laser cutting of different polymeric plastics (PE, PP and PC) by a CO2 laser beam” J. Materials Processing Technology 159 279-285.

A. Folch, Introduction to BioMEMS (Academic Press, 2010).

T-.W. Lee [2008], Thermal and Flow Measurements (CRC Press, Boca Raton, Florida).

A.McAndrew [2004], Introduction to Digital Image Processing with MATLAB® (Thompson).

N.-T. Nguyen and S.T. Werely, Fundamentals and Applications of Microfluidics (Artech House, Norwood, MA).

A.Rios, A. Escarpa, and B. Simonet [2009] Miniaturization of Analytical Systems (Wiley).

P. Tableling, Introduction to Microfluidics (Oxford University Press, 2005)

G.M. Whitesides [2006], “The Origins and Future of Microfluidics” Nature 442 368-373. 